TRANSACTIONS OF THE

AMERICAN MATHEMATICAL SOCIETY

Volume 351, Number 2, February 1999, Pages 559-568

$\mathrm{S} 0002-9947(99) 02383-1$

\title{
REGULARITY OF SOLUTIONS TO THE MEASURABLE LIVSIC EQUATION
}

\author{
M. POLLICOTT AND M. YURI
}

\begin{abstract}
In this note we give generalisations of Livsic's result that a priori measurable solutions to cocycle equations must in fact be more regular. We go beyond the original continuous hyperbolic examples of Livsic to consider examples of this phenomenon in the context of:

(a) $\beta$-transformations;

(b) rational maps; and

(c) planar maps with indifferent periodic points.

Such examples are not immediately covered by Livsic's original approach either due to a lack of continuity or hyperbolicity.
\end{abstract}

\section{INTRODUCTION}

In 1972 Livsic showed that given a mixing subshift of finite type $T: X \rightarrow X$ and a Hölder continuous function $c: X \rightarrow \mathbb{R}$ then any solution to the equation

$$
c(x)=u(T x)-u(x)
$$

with $u: X \rightarrow \mathbb{R}$ measurable and essentially bounded (with respect to an equilibrium measure for a Hölder continuous function) must have a continuous version i.e. $\exists u^{\prime}$ : $X \rightarrow \mathbb{R}$ continuous such that $u(x)=u^{\prime}(x)$ a.e. [2], [3]

This is an elementary type of "rigidity result". Livsic also applied this result to Anosov systems. Another approach to this result for subshifts of finite type and using Perron-Frobenius type operators appeared in [6].

In this note we shall discuss some simple generalisations of Livsic's result to other examples of dynamical systems. We shall be interested in systems with discontinuities or which are not uniformly hyperbolic and thus are not covered by Livsic's original results. In this context the Perron-Frobenius type operator method adapts easily to prove the generalisations of Livsic's original results.

To illustrate this approach we shall apply this method to $\beta$-transformations (section 1), rational maps (section 2) and certain multi-dimensional maps (section $3)$.

\section{1. $\beta$-TRANSFORMATION}

We begin by recalling the definition of the well-known $\beta$-transformation on the unit interval. Let $\beta>1$ and define $T:[0,1) \rightarrow[0,1)$ by $T(x)=\beta x(\bmod 1)$. Let $\nu$ denote Lebesgue measure on the interval $[0,1]$ and $L^{1}([0,1], \mathcal{B}, \nu)$ denotes the space of integrable functions on $[0,1]$.

Received by the editors August 5, 1996.

1991 Mathematics Subject Classification. Primary 58Fxx.

(C)1999 American Mathematical Society 
Definition. We define a Perron-Frobenius operator

$$
\mathcal{L}_{1}: L^{1}([0,1], \mathcal{B}, \nu) \rightarrow L^{1}([0,1], \mathcal{B}, \nu)
$$

by

$$
\mathcal{L}_{1} f(x)=\sum_{T y=x} \frac{1}{\beta} f(y) .
$$

where the summation is over the $[\beta]$ or $[\beta]+1$ pre-images of $x$.

We denote by $B_{B V}$ the space of functions of bounded variation i.e. if $f \in B_{B V}$ then we have that

$$
\operatorname{var}(f):=\sup \left\{\sum_{i=0}^{n-1}\left|f\left(x_{i+1}\right)-f\left(x_{i}\right)\right|: 0=x_{0}<x_{1}<\ldots<x_{n}=1\right\}<+\infty .
$$

It is easy to check that the operator $\mathcal{L}_{1}$ preserves $B_{B V}$.

The following result is due to W. Parry [5].

Lemma 1. The function $h:[0,1] \rightarrow \mathbb{R}$ defined by

$$
h(x)=\sum_{T^{n}(1) \in(x, 1]} \frac{1}{\beta^{n}}
$$

satisfies $\mathcal{L}_{1} h(x)=h(x)$. In particular, $h \in B_{B V}$ and $h>0$ for $x \in[0,1)$.

We shall also need the following more general operator.

Definition. Given a Lipschitz function $c:[0,1] \rightarrow \mathbb{R}$. We define a $c$-weighted Perron-Frobenius operator $\mathcal{L}_{2}: L^{1}([0,1], \mathcal{B}, \nu) \rightarrow L^{1}([0,1], \mathcal{B}, \nu)$ by

$$
\mathcal{L}_{2} f(x)=\sum_{T y=x} \frac{e^{c(y)}}{\beta} f(y) .
$$

(When $c=0$ then $\mathcal{L}_{2}$ clearly reduces to $\mathcal{L}_{1}$.)

It is convenient to denote $c^{n}(x)=c(x)+c(T x)+\ldots+c\left(T^{n-1} x\right)$ for $n \geq 1$. The following result is important in proving Theorem 1.

Lemma 2. (i) $\forall f \in L^{1}([0,1], \mathcal{B}, \nu)$ we have $\mathcal{L}_{1}^{n} f \rightarrow h\left(\int f d \nu\right)$ in the $L^{1}$ norm,

(ii) Let $c:[0,1] \rightarrow \mathbb{R}$ be a Lipschitz function. There exists $w \in B_{B V}$ with $w>0$ and $\lambda>0$ such that the sequence of functions

$$
f_{n}(x)=\frac{1}{\lambda^{n}}\left(\sum_{T^{n} y=x} \frac{e^{c^{n}(y)}}{\beta^{n}}\right)
$$

converges to $w$ in the $L^{1}$ norm.

Proof. The proof makes use of a number of results from [9].

(i) It is easy to check that $\mathcal{L}_{1}: B_{B V} \rightarrow B_{B V}$. The convergence

$$
\mathcal{L}_{1}^{n} f \rightarrow h\left(\int f d \nu\right), \quad \text { as } n \rightarrow+\infty
$$

is proved in [9, Theorem 10] when $f$ is uniformly continuous. Since any function in $L^{1}([0,1], \mathcal{B}, \nu)$ can be approximated from above and below by such functions, this is sufficient. 
(ii) The operator $\mathcal{L}_{2}: B_{B V} \rightarrow B_{B V}$ has a simple maximal eigenvalue $\lambda$, say, with positive eigenvector $w \in B_{B V}([9$, Lemma 9]). Moreover, there exists a measure $\mu$ on $[0,1]$ such that

$$
f_{n}=\frac{\mathcal{L}_{2}^{n} f}{\lambda^{n}} \rightarrow w \int f d \mu, \quad \text { as } n \rightarrow+\infty
$$

[9, Theorem 10].

Our main result on the measurable Livsic equation for $\beta$-transformations is the following.

Theorem 1. If $c:[0,1] \rightarrow \mathbb{R}$ is Lipschitz then for any measurable and essentially bounded solution $u$ to

$$
c(x)=u(T x)-u(x)
$$

there exists $u_{0}(x)=v(x)-\log h(x)$, where $v(x) \in B_{B V}$ such that $u=u_{0}$ a.e. $(\mu)$.

Proof. We can make the choice $f=1$ and then iterating the operator $\mathcal{L}_{2}$ introduced above we can write

$$
\left(\mathcal{L}_{2}^{n} 1\right)(x)=\sum_{T^{n} y=x} \frac{e^{c^{n}(y)}}{\beta^{n}}=\lambda^{n} f_{n}(x)
$$

In particular, substituting from the identity $\left(1^{\prime}\right)$ we get that

$$
\begin{aligned}
f_{n}(x) & =\frac{1}{\lambda^{n}} \sum_{T^{n} y=x} \frac{e^{c^{n}(y)}}{\beta^{n}} \\
& =\frac{1}{\lambda^{n}} \sum_{T^{n} y=x} \frac{e^{\left[u\left(T^{n} y\right)-u(y)\right]}}{\beta^{n}} \\
& =\frac{e^{u(x)}}{\lambda^{n}}\left(\sum_{T^{n} y=x} \frac{e^{-u(y)}}{\beta^{n}}\right) \\
& =\frac{e^{u(x)}}{\lambda^{n}}\left(\mathcal{L}_{1}^{n} e^{-u}\right)(x) \quad \text { a.e. }
\end{aligned}
$$

By part (ii) of Lemma 2 we see that

$$
\lim _{n \rightarrow+\infty} f_{n}(x)=w(x) \quad \text { a.e. }
$$

Applying part (i) of Lemma 2 with the choice $f(x)=e^{-u(x)}$ gives that

$$
\lim _{n \rightarrow+\infty} e^{u(x)}\left(\mathcal{L}_{1}^{n} e^{-u}\right)(x)=e^{u(x)} h(x)\left(\int e^{-u} d \nu\right) \quad \text { a.e. }
$$

Taking the limit $n \rightarrow+\infty$ in (2) allows us to conclude that

(a) $\lambda=1$ (since the limits (3) and (4) are finite and non-zero), and

(b) $w(x)=e^{u(x)} h(x)\left(\int e^{-u} d \nu\right)$ a.e.

Taking logarithms in (b) and writing $v(x)=\log w(x)$ gives the result.

The following corollary is immediate.

Corollary. There is a solution $u_{0}(x)$ to $\left(1^{\prime}\right)$ which for any $\epsilon>0$ is of bounded variation on the subinterval $[0,1-\epsilon]$. 


\section{RATiONAL MAPS}

Let $\hat{\mathbb{C}}$ denote the extended complex plane and let $f: \hat{\mathbb{C}} \rightarrow \hat{\mathbb{C}}$ denote a rational map $f(z)=\frac{P(z)}{Q(z)}$, where $P, Q \in \mathbb{R}[z]$ are polynomials which have no common divisors. We shall also assume that the degree $d=\max (\operatorname{deg}(P), \operatorname{deg}(Q)) \geq 2$. Let $J$ denote the Julia set for the rational map (i.e. the closure of the repelling periodic points).

There is a natural measure on $J$ defined by the following construction.

Proposition 1 (Lyubich [4]). There exists a probability measure $\mu$ on $J$ such that for any point $x \in J$ we have the converence

$$
\frac{1}{d^{n}} \sum_{T^{n} y=x} \delta_{y} \rightarrow \mu \quad \text { as } n \rightarrow+\infty
$$

(in the weak ${ }^{*}$ topology) where $\delta_{y}$ denotes the Dirac measure supported on $y \in J$.

We can introduce an operator on the continuous functions on $J$ defined as follows.

Definition. We define the operator $\mathcal{L}: C^{0}(J) \rightarrow C^{0}(J)$ by

$$
\mathcal{L}_{1} f(x)=\sum_{T y=x} \frac{f(y)}{d}
$$

where the sum is over the $d$ pre-images of $T$ repeated according to multiplicity.

It is easy to see that $\mathcal{L}_{1} 1=1$. Given $c \in C^{0}(J)$ we recall that the pressure of $c$ is defined by

$$
P(c)=\sup \left\{h_{\mu}(T)+\int c d \mu: \mu \text { is a } T \text {-invariant probability }\right\} .
$$

For $\alpha>0$ we let $C^{\alpha}(J)$ denote the Hölder continuous functions on $J$.

Lemma 3. Let $c: J \rightarrow \mathbb{R}$ be an element of $C^{\alpha}(J)$ such that $P(c)>\sup _{x \in J} c(x)$. There exists $w \in C^{0}(J)$ with $w>0$ and $\lambda>0$ such that the sequence of functions

$$
f_{n}(x)=\frac{1}{\lambda^{n}}\left(\sum_{T^{n} y=x} \frac{e^{c^{n}(y)}}{d^{n}}\right)
$$

converges to $w$ (almost everywhere).

Proof. By choosing $\alpha>0$ smaller, if necessary, we can consider the operator $\mathcal{L}_{2}$ : $C^{\alpha}(J) \rightarrow C^{\alpha}(J)$ defined by

$$
\left(\mathcal{L}_{2} f\right)(x)=\frac{1}{d} \sum_{T y=x} e^{c(y)} f(y) .
$$

This operator has a simple maximal eigenvalue $\lambda=e^{P(c)} / d$ with maximal eigenvector $w>0$ [7]. Furthermore,

$$
f_{n}(x)=\frac{\left(\mathcal{L}_{2}^{n} 1\right)(x)}{\lambda^{n}} \rightarrow w, \quad \text { as } n \rightarrow+\infty
$$

with convergence in the supremum norm [7].

Our main result for rational maps takes the following form. 
Theorem 2. If $c \in C^{\alpha}(J)$ with $P(c)>\sup _{x \in J} c(x)$ and $u$ is a measurable essentially bounded solution to

$$
c(x)=u(T x)-u(x),
$$

then there exists $u_{0}(x) \in C^{0}(J)$ such that $u=u_{0}$ a.e. $(\mu)$.

Proof. The proof is similar to that of Theorem 1. We can write

$$
\left(\mathcal{L}_{2}^{n} 1\right)(x)=\sum_{T^{n} y=x} \frac{e^{c^{n}(y)}}{d^{n}}
$$

and then substituting from the identity $\left(1^{\prime \prime}\right)$ we get that

$$
\begin{aligned}
f_{n}(x) & =\frac{1}{\lambda^{n}}\left(\mathcal{L}_{2}^{n} 1\right)(x) \\
& =\frac{e^{u(x)}}{\lambda^{n}}\left(\sum_{T^{n} y=x} \frac{e^{-u(y)}}{d^{n}}\right) \\
& =\frac{e^{u(x)}}{\lambda^{n}}\left(\mathcal{L}_{1}^{n} e^{-u}\right)(x) \quad \text { a.e. }
\end{aligned}
$$

By Proposition 1 we have for all $x \in J$ :

$$
\lim _{n \rightarrow+\infty} \mathcal{L}_{1}^{n}\left(e^{-u}\right)(x)=\int e^{-u(x)} d \mu(x)
$$

and by Lemma 3

$$
\lim _{n \rightarrow+\infty} f_{n}(x)=w(x) \quad \text { a.e. }
$$

Comparing (5), (6) and (7) we have:

(a) $\lambda=1$ (since the limits in (6) and (7) are both finite and non-zero); and

(b) $w(x)=e^{u(x)} h(x)\left(\int e^{-u} d \mu\right)$ a.e.

Taking logarithms in (b) gives the required result.

Remarks. If we assume that that $J$ is a hyperbolic Julia set (i.e. $\inf _{z \in J}\left|T^{\prime}(z)\right|>1$ ) then it is easy to see that the hypothesis $P(c)>\sup _{x \in J} c(x)$ is no longer required and the function $w$ can be shown to be Hölder continuous.

\section{MAPS ON SUBSETS OF $\mathbb{R}^{d}$}

Let $X$ be a subset of $\mathbb{R}^{d}$ and let $\nu$ denote the (normalised) Lebesgue measure on $X$. Let $T: X \rightarrow X$ be a non-singular transformation. We shall want to restrict to non-uniformly hyperbolic systems $T: X \rightarrow X$ which are piecewise $C^{1}$-invertible and admit indifferent periodic orbits.

Definition. We call $\left(T, X, Q=\left\{X_{a}\right\}_{a \in I}, \mathcal{U}\right)$ a piecewise invertible system with Finite Range structure (FRS) if $T$ is a map on a bounded domain $X \subset \mathbb{R}^{d}, Q=$ $\left\{X_{a}\right\}_{a \in I}$ is a countable generating partition of $X$ and $\mathcal{U}$ is a collection of finitely many subsets (not necessarily disjoint) with positive Lebesgue measure of $X$, such that

1. each $X_{a}$ is a measurable connected subset with piecewise smooth boundary and $\operatorname{int}\left(X_{a}\right) \neq \emptyset$,

2. for each $X_{a},\left.T\right|_{X_{a}}$ is injective, of class $C^{1}$ with $\left.\operatorname{det} D T\right|_{X_{a}} \neq 0$, 
3. Denote the cylinder $X_{a_{1}} \cap T^{-1} X_{a_{2}} \cap \ldots \cap T^{-(n-1)} X_{a_{n}}$ by $X_{a_{1} \ldots a_{n}}$ if its interior is not empty. Then $T^{n} X_{a_{1} \ldots a_{n}} \in \mathcal{U}$ (the FRS-condition).

Typical examples are number theoretical transformations. (Cf. [1], [10]-[12].) In the special case that $\mathcal{U} \subset Q$ this is a Markov map. However, in general we will not make this assumption. The following simple example satisfies all of these hypotheses.

Example. We shall describe a real two-dimensional map which is related to a complex continued fraction expansion. Let $\alpha=1+i$ then we denote

$$
X=\left\{z=x_{1} \alpha+x_{2} \bar{\alpha}:-(1 / 2) \leq x_{1}, x_{2} \leq 1 / 2\right\}
$$

and define $T: X \rightarrow X$ by

$$
T(z)=1 / z-[1 / z]_{1},
$$

where $[z]_{1}$ denotes $\left[x_{1}+1 / 2\right] \alpha+\left[x_{2}+1 / 2\right] \bar{\alpha}$ for a complex number $z=x_{1} \alpha+x_{2} \bar{\alpha}$ (and [.] denotes the integar part). Let $I=\{n \alpha+m \bar{\alpha}: m, n \in \mathbb{Z}\} \backslash\{0\}$. For each $n \alpha+m \bar{\alpha} \in I$, we define

$$
X_{n \alpha+m \bar{\alpha}}=\left\{z \in X:[1 / z]_{1}=n \alpha+m \bar{\alpha}\right\} .
$$

Then we have a countable partition $Q=\left\{X_{a}\right\}_{a \in I}$ of $X$. The map $T$ induces a continued fraction like expansion of $z \in X$,

$$
z=\frac{1}{a_{1}+\frac{1}{a_{2}+\frac{1}{\cdots \frac{1}{a_{n}+\ldots}}}},
$$

where each $a_{i}$ is contained in $I$. The family $\mathcal{U}$ consists of 5 subsets of $X$ and the map $T$ has indifferent periodic points $\pm 1, \pm i$ and the invariant density which was obtained by Tanaka [8] has singulalities at these point and no others. (The Weak Bernoulli property and further dynamical properties are discussed in [10]-[11], [13].)

Let $\mathcal{V}$ denote the disjoint partition generated by $\mathcal{U}$. Let $D_{k}$ denote the union of cylinders $X_{a_{1} \ldots a_{k}}$ of rank $k$ which touch indifferent periodic points. We let $D_{k}^{c}$ denote its complement. (In particular, we shall use the convention $D_{0}=X$.)

Remark. Typically, the intersection $\bigcap_{n=0}^{\infty} D_{n}$ is merely the set of indifferent periodic orbits.

Definition. For a function $\phi: X \rightarrow \mathbb{R}$, we define the generalized Perron-Frobenius operator $\mathcal{L}_{\phi}: L^{1}(X, \nu) \rightarrow L^{1}(X, \nu)$ by

$$
\mathcal{L}_{\phi} f(x)=\sum_{y \in T^{-1} x} e^{\phi(y)} f(y)
$$

We denote $\mathcal{L}_{1}=\mathcal{L}_{-\log |\operatorname{det} D T|}$ and recall the following result $[13$, Theorems 3.1-3.2].

Lemma 4. There exists a piecewise continuous function $h>0$ such that $\mathcal{L}_{1} h(x)=$ $h(x)$. Futhermore, for any $f \in C(\bar{X})$

$$
\mathcal{L}_{1}^{n} f \rightarrow\left(\int f d \nu\right) h \text { as } n \rightarrow \infty
$$

with uniform converge on compact subsets of $\left(X-\bigcap_{n=0}^{\infty} D_{n}\right) \cap(X-\partial \mathcal{V})$, (i.e., on any compact subset of $X$ excluding $\bigcap_{n=0}^{\infty} D_{n}$ and the boundary of range sets $U \in \mathcal{U}$.) 
We need to make the following additional two assumptions on the function $c$ :

(C-1) For each $k>0$,

$$
\gamma_{1}(k)=\sup _{x \in D_{k}^{c}\left(a_{1} \ldots a_{n}\right): T^{n} X_{a_{1} \ldots a_{n}} \ni x}\left(\exp \left[\sum_{i=1}^{n} c\left(\psi_{a_{i} \ldots a_{n}}(x)\right)\right]\right)<\infty
$$

where $\psi_{a_{i} \ldots a_{n}}: T^{n-i+1} X_{a_{i} \ldots a_{n}} \rightarrow X_{a_{i} \ldots a_{n}}$ is the local inverse to $T^{n-i+1}$; and (C-2) For each $k>0, \exists 0<\gamma_{2}(k)<\infty \forall x, x^{\prime} \in D_{k}^{c} \cap V$ (with $V \in \mathcal{V}$ ),

$$
\sup _{\left(a_{1} \ldots a_{n}\right): T^{n} X_{a_{1} \ldots a_{n}} \ni x, x^{\prime}}\left|1-\frac{\exp \left(\sum_{i=1}^{n} c\left(\psi_{a_{i} \ldots a_{n}} x\right)\right)}{\exp \left(\sum_{i=1}^{n} c\left(\psi_{a_{i} \ldots a_{n}} x^{\prime}\right)\right)}\right| \leq \gamma_{2}(k)\left\|x-x^{\prime}\right\| .
$$

We denote $\mathcal{L}_{2}=\mathcal{L}_{c-\log |\operatorname{det}(D T)|}$.

Lemma 5. Assume that c satisfies (C-1) and (C-2).

(i) Then there is a sequence $n_{j} \rightarrow+\infty$ as $j \rightarrow \infty$ and a locally Lipschitz function $\omega$ such that

$$
f_{n_{j}}(x)=\frac{1}{n_{j}} \sum_{i=0}^{n_{j}-1} \mathcal{L}_{2}^{i}(1) \rightarrow \omega, \text { as } j \rightarrow \infty
$$

uniformly on any compact subset of $\left(X-\bigcap_{n=0}^{\infty} D_{n}\right) \cap(X-\partial \mathcal{V})$; and

(ii) we have also the convergence almost everywhere

$$
\frac{1}{n_{j}} \sum_{i=0}^{n_{j}-1} \mathcal{L}_{1}^{i}\left(e^{-u}\right) \rightarrow \frac{\omega}{e^{u}}, \text { as } j \rightarrow \infty
$$

Proof. To prove part (i) we first want to establish the boundedness and equicontinuity of $\left\{\mathcal{L}_{2}^{n}(1)\right\}_{n>0}$ on compact subsets of $\left(X-\bigcap_{n=0}^{\infty} D_{n}\right) \cap(X-\partial \mathcal{V})$. We begin by observing that $\left\{\mathcal{L}_{1}^{n}(1)\right\}$ is bounded in the supremum norm restricted on $D_{k}^{c}$. To see this, we consider a third Perron Frobenius operator $\mathcal{L}_{3}=\mathcal{L}_{\phi}$ for $\phi(x)=-\log |\operatorname{det} D T(x)|+\log h(x)-\log h(T x)$. It is easy to see that for each $n>0$ we can write

$$
\mathcal{L}_{3}^{n} f(x)=h(x)^{-1} \mathcal{L}_{1}^{n}(f h)(x)
$$

In particular, we have that $\mathcal{L}_{1}^{n}(1)=h \mathcal{L}_{3}^{n}\left(h^{-1}\right)$. However, since $h$ is bounded from above on $D_{k}^{c}$ (in Lemma 4 ) and from below on $X$ (by Remark $\mathrm{F}$ in [13, p.983]), we have the boundedness of $\mathcal{L}_{1}^{n}(1)$ restricted to $D_{k}^{c}$.

We claim that for each $k>0, \exists 0<\gamma_{3}(k)<\infty$ such that

$$
\left|\mathcal{L}_{2}^{n}(1)(x)-\mathcal{L}_{2}^{n}(1)\left(x^{\prime}\right)\right| \leq \gamma_{3}(k)\left\|x-x^{\prime}\right\|, \quad \forall x, x^{\prime} \in D_{k}^{c} \cap V,(V \in \mathcal{V})
$$


To see this we write

$$
\begin{aligned}
&\left|\mathcal{L}_{2}^{n}(1)(x)-\mathcal{L}_{2}^{n}(1)\left(x^{\prime}\right)\right|\left|\sum_{y \in T^{-n} x} \frac{e^{c^{n}(y)}}{\left|\operatorname{det} D T^{n}(y)\right|}-\sum_{y^{\prime} \in T^{-n} x^{\prime}} \frac{e^{c^{n}\left(y^{\prime}\right)}}{\left|\operatorname{det} D T^{n}\left(y^{\prime}\right)\right|}\right| \\
&=\left|\sum_{\substack{\left(a_{1}, \ldots, a_{n}\right): \\
x \in T^{n} X_{a_{1} \ldots a_{n}}}} \exp \left(\sum_{i=1}^{n} c\left(\psi_{a_{i} \ldots a_{n}} x\right)\right)\right| \operatorname{det} D \psi_{a_{1} \ldots a_{n}}(x) \mid \\
& \quad-\sum_{\substack{\left(a_{1}, \ldots, a_{n}\right): \\
x^{\prime} \in T^{n} X_{a_{1} \ldots a_{n}}}} \exp \left(\sum_{i=1}^{n} c\left(\psi_{a_{i} \ldots a_{n}} x^{\prime}\right)\right)\left|\operatorname{det} D \psi_{a_{1} \ldots a_{n}}\left(x^{\prime}\right)\right| \mid \\
& \leq\left|\sum_{\substack{\left(a_{1}, \ldots, a_{n}\right): \\
T^{n} X_{a_{1} \ldots a_{n}} \ni x, x^{\prime}}} \exp \left(\sum_{i=1}^{n} c\left(\psi_{a_{i} \ldots a_{n}} x\right)\right)\right| \operatorname{det} D \psi_{a_{1} \ldots a_{n}}(x) \mid \\
&\left.+\sum_{\substack{\left(a_{1}, \ldots, a_{n}\right): \\
T^{n} X_{a_{1} \ldots a_{n}} \ni x, x^{\prime}}} \times\left(1-\frac{\left|\operatorname{det} D \psi_{a_{1} \ldots a_{n}}\left(x^{\prime}\right)\right|}{\left|\operatorname{det} D \psi_{a_{1} \ldots a_{n}}(x)\right|}\right)\right) \mid \\
& \quad \times\left(1-\frac{\operatorname{det}\left(D \psi_{a_{i} \ldots a_{n}}\left(x^{\prime}\right) \mid \exp \left(\sum_{i=1}^{n} c\left(\psi_{a_{i} \ldots a_{n}} x\right)\right)\right.}{\exp \left(\sum_{i=1}^{n} c\left(\psi_{a_{i} \ldots a_{n}} x^{\prime}\right)\right)}\right) \mid .
\end{aligned}
$$

From (C-1),(C-2) and (E-1) in [13], we have immediately the desired result. It follows that

$$
\left\{\frac{1}{n} \sum_{i=0}^{n-1} \mathcal{L}_{2}^{i}(1)\right\}_{n=0}^{\infty}
$$

is also bounded in the supremum norm and piecewise equicontinuous on $D_{k}^{c} \cap V$ for each $k>0$ and $V \in \mathcal{V}$ and so contains a convergent subsequence. We now prove part (ii). By the definition of $\mathcal{L}_{1}$ and $\mathcal{L}_{2}$ we have that

$$
\begin{aligned}
\mathcal{L}_{2}{ }^{n}(1) & =\mathcal{L}_{c-\log |\operatorname{det}(D T)|}^{n}(1) \\
& =e^{u} \mathcal{L}_{1}{ }^{n}\left(e^{-u}\right)
\end{aligned}
$$

for $n \geq 0$. Thus (ii) follows immediately from (i)

Lemma 6. Assume that $u$ is measurable and essentially bounded. Then

$$
\mathcal{L}_{1}\left(\frac{\omega}{e^{u}}\right)=\frac{\omega}{e^{u}} \quad \text { a.e. }
$$


Proof. Observe that

$$
\begin{aligned}
\mathcal{L}_{1}\left(\frac{1}{n_{j}} \sum_{i=0}^{n_{j}-1} \mathcal{L}_{1}^{i}\left(e^{-u}\right)\right) & =\frac{1}{n_{j}} \sum_{i=1}^{n_{j}} \mathcal{L}_{1}^{i}\left(e^{-u}\right) \\
& =\frac{1}{n_{j}}\left\{\sum_{i=0}^{n_{j}} \mathcal{L}_{1}^{i}\left(e^{-u}\right)-e^{-u}\right\} \\
& =\frac{1}{n_{j}} \sum_{i=0}^{n_{j}} \mathcal{L}_{1}^{i}\left(e^{-u}\right)\left\{1-\frac{e^{-u}}{\sum_{i=0}^{n_{j}} \mathcal{L}_{1}^{i}\left(e^{-u}\right)}\right\} .
\end{aligned}
$$

Then

$$
\sum_{i=0}^{n_{j}} \mathcal{L}_{1}^{i}\left(e^{-u}\right) \geq \sum_{i=0}^{n_{j}} \exp \left(-\|u\|_{\infty}\right) \sum_{a_{1} \ldots a_{i}}\left|\operatorname{det} D \psi_{a_{1} \ldots a_{i}}(x)\right| \chi_{T^{i} X_{a_{1} \ldots a_{i}}}(x)
$$

where $\chi_{T^{i} X_{a_{1} \ldots a_{i}}}$ is the characteristic function for the set $T^{i} X_{a_{1} \ldots a_{i}}$. It follows from Lemma 4 that $\mathcal{L}_{1}^{n}(1) \rightarrow h$ uniformly on a compact subset of $\bigcup_{i=1}^{\infty} B_{i} \cap V(V \in \mathcal{V})$. From this and the fact $h \geq G^{-1}$ (cf. [1], [10]) for some constant $0<G<\infty$, we conclude that $\sum_{i=0}^{n_{j}} \mathcal{L}_{1}^{i}(1) \geq n_{j} G^{-1} \rightarrow+\infty$ as $j \rightarrow+\infty$.

Hence by Lemma 5 (ii) we have

$$
\lim _{j \rightarrow \infty} \mathcal{L}_{1}\left(\frac{1}{n_{j}} \sum_{i=0}^{n_{j}-1} \mathcal{L}_{1}^{i}\left(e^{-u}\right)\right)=\lim _{j \rightarrow \infty} \frac{1}{n_{j}+1} \sum_{i=0}^{n_{j}} \mathcal{L}_{1}^{i}\left(e^{-u}\right)=\frac{\omega}{e^{u}}
$$

This completes the proof of Lemma 6 .

Lemma 7. For each $k>0$ and $V \in \mathcal{V}$ the function $h$ is piecewise Lipschitz continuous on $D_{k}^{c} \cap V$.

Proof. The result follows from Lemma 3.2 and the proof of Theorem 3.1 in [13].

Thus we come to the main result

Theorem 3. If there exists a measurable and essentially bounded solution $u$ to

$$
c(x)=u(T x)-u(x)
$$

and the function c satisfies (C-1),(C-2), then there exists a piecewise Lipschitz solution $u_{0}$ piecewise Lipschitz continuous on compact subset of $\left(X-\bigcap_{n=0}^{\infty} D_{n}\right) \cap$ $(\partial \mathcal{V})^{c}$ and $u=u_{0}$ (a.e.)

Proof. There is a unique fixed point $h$, say, for $\mathcal{L}_{1}: L^{1}(X, \nu) \rightarrow L^{1}(X, \nu)$ and

$$
\mathcal{L}_{1}^{n}\left(e^{-u}\right) \rightarrow(\text { constant }) h
$$

since the invariant measure $d \mu=h d \nu$ obtained is ergodic and is equivalent to the Lebesgue measure $\nu$ (cf. [1]). By Lemma 4 and Lemma 6 . we have the equation:

$$
\frac{\omega}{e^{u}}=\text { (constant) } h \text { (a.e.) }
$$

This completes the proof. 


\section{REFERENCES}

1. Sh.Ito and M.Yuri, Number theoretical transformations with finite range structure and their ergodic properties, Tokyo J.Math 10 (1987), 1-32. MR 88i:28031

2. A. Livsic, Homology properties of $Y$-systems, Math. Zametki 10 (1971), 758-763.

3. A. Livsic, Cohomology of dynamical systems, Math. USSR Izvestija 6 (1972), 1278-1301. MR 48: 12606

4. M. Lyubich, Ergodic properties of rationale endomorphisms of the Riemann sphere, Ergod. Theory and Dynam. Sys. 3 (1983), 351-385.

5. W. Parry, Acta Math. Acad. Sci. Hungary 11 (1960), 401-416. MR 26:288

6. W. Parry and M. Pollicott, Zeta functions and the closed orbit structure of hyperbolic systems, Asterisque 187-188 (1990), 1-267. MR 92f:58141

7. F. Przytycki, On the Perron-Frobenius-Ruelle operator for rational maps on the Riemann sphere and for Hölder continuous functions, Bol. Bras. Math. Soc. 20 (1990), 95-125. MR 93b:58120

8. S.Tanaka, A complex continued fraction and its ergodic properties, Tokyo J.Math. 8 (1985), 191-214. MR 87h:11073

9. P. Walters, Equilibtium States for $\beta$-Transformations and Related Transformations, Math. Z. 159 (1978), 65-88. MR 57:6370

10. M.Yuri, On a Bernoulli property for multi-dimensional maps with finite range structure, Tokyo J.Math 9 (1986), 457-485. MR 88d:28023

11. M.Yuri, Invariant measures for certain multi-dimensional maps, Nonlinearity 7 (1994), 10931124. MR 95c: 11101

12. M.Yuri, Multi-dimensional maps with infinite invariant measures and countable state sofic shifts, Indagationes Mathematicae 6 (1995), 355-383. MR 96f:28021

13. M.Yuri, On the convergence to equilibrium states for certain nonhyperbolic systems., Ergodic Theory and Dynam.Sys. 17 (1997), 977-1000. MR 98f:58155

Department of Mathematics, Manchester University, Manchester, M13 9PL, EngLAND

E-mail address: mp@ma.man.ac.uk

Department of Business Administration, Sapporo University, 3-Jo, 7-Chome, Nishioka, TOYOHIRA-KU, SAPPORO 062, JAPAN

E-mail address: yuri@math.sci.hokudai.ac.jp 